\title{
Subacute combined degeneration of the spinal cord masking motor neuron disease: a case report
}

\author{
Paula Loveland ${ }^{1 *} \mathbb{D}$, Aaron Wong ${ }^{2}$, Vinojini Vivekanandam ${ }^{3}$ and Wen Kwang Lim ${ }^{1,4}$
}

\begin{abstract}
Background: Subacute combined degeneration of the spinal cord is a potentially reversible myelopathy typically associated with vitamin B12 deficiency. There is predominant involvement of spinal cord posterior and lateral tracts, and manifestations include peripheral paraesthesia, impaired proprioception, gait disturbance, neuropathy and cognitive changes. Motor neuron disease (MND) is an unremittingly progressive neurodegenerative disorder involving upper and lower motor neurons with an average prognosis of 2-3 years. The diagnosis is clinical and may be supported by electromyography. A subset of MND occurs concurrently with frontotemporal dementia (FTDMND) and may be initially misdiagnosed as a primary psychotic disorder.

Case presentation: We describe a 57-year-old Caucasian woman who presented with confusion and dysarthria. Low vitamin B12 levels and MRI findings led to an initial diagnosis of subacute combined degeneration of the spinal cord. Despite treatment, persistent dysarthria and presence of both upper and lower motor neuron signs on clinical examination prompted further assessment. Electromyography supported the diagnosis of MND. Comorbid chronic paranoid schizophrenia complicated the diagnostic process. We discuss overlapping features between B12 deficiency and MND as well as the neuropsychiatric overlap of B12 deficiency, FTD-MND and chronic schizophrenia.

Conclusions: Firstly, variability in neurocognitive and imaging manifestations of B12 deficiency can limit delineation of other pathologies. Failure to improve following correction of nutritional deficiencies warrants further investigation for an alternate diagnosis. Secondly, re-evaluation of patients with comorbid mental health conditions is important in reaching timely and accurate diagnoses.
\end{abstract}

Keywords: Vitamin B 12, subacute combined degeneration, motor neuron disease, schizophrenia

\section{Background}

Subacute combined degeneration of the spinal cord is a potentially reversible myelopathy typically associated with vitamin B12 deficiency. There is predominant involvement of spinal cord posterior and lateral tracts, and manifestations include peripheral paraesthesia, impaired proprioception, gait disturbance, neuropathy and cognitive changes [1]. Motor neuron disease (MND), its commonest subtype being amyotrophic lateral sclerosis (ALS), is an unremittingly progressive neurodegenerative disorder involving upper and lower motor neurons with an average prognosis of two to three years [2]. The diagnosis is

\footnotetext{
*Correspondence: paula.loveland@mh.org.au

'Department of Medicine and Aged Care, The Royal Melbourne Hospital,

Parkville, VIC, Australia

Full list of author information is available at the end of the article
}

clinical and may be supported by electromyography (EMG). Neuroimaging findings in both conditions are insensitive, often unremarkable, or as rarely described they may be similar or even indistinguishable $[3,4]$. We highlight the diagnostic challenges posed by coexistence of vitamin B12 deficiency and another neurological pathology, particularly MND, as there are overlapping clinical features common to both conditions.

The importance of a high index of suspicion in patients with cognitive and psychiatric conditions is also reinforced by our case, as such patients are vulnerable to missed or delayed diagnoses. 'Diagnostic overshadowing', or misattribution of disease manifestations to that of a known psychiatric condition, is commonly described in the literature, as are atypical disease presentations due to a variety of factors [5]. 
We also discuss our patient's case in light of the subset of MND patients that manifest psychotic symptoms as part of the MND with frontotemporal dementia (FTDMND) syndrome, of which the C9ORF72 mutation is the commonest abnormality in familial cases. Recent genetic studies also suggest a possible link between MND and chronic psychosis without dementia.

\section{Case presentation}

A 57-year-old Caucasian woman with a background of treatment-resistant schizophrenia for over 30 years, stable on clozapine, was admitted after being found confused, dysarthric and dehydrated at home, having been uncontactable for several days. Her medical history included unprovoked pulmonary emboli on warfarin and emphysema, and her family history was significant for one brother with schizophrenia and one unaffected sister, but no known family members with MND or dementia-type diagnoses.

Confusion improved following hydration, but she was unable to recall recent events. Paranoia and distrust of staff were consistent with her chronic delusions and limited engagement with assessments. Neurological examination revealed tongue atrophy, increased upper and lower limb tone with hyperreflexia bilaterally, left upper limb and lower limb weakness and proprioception deficits without ataxia.
On admission, full blood examination, electrolytes and renal function were normal. Creatinine kinase was elevated at $882 \mathrm{IU} / \mathrm{L}$ (reference: $30-70 \mathrm{IU} / \mathrm{L}$ ), vitamin B12 level was low at $69 \mathrm{pmol} / \mathrm{L}(140-650 \mathrm{pmol} / \mathrm{L})$ and folate was normal at $31.5 \mathrm{nmol} / \mathrm{L} \quad(>7.0 \mathrm{nmol} / \mathrm{L})$. Computed tomography (CT) scan of the head, MRI diffusion weighted imaging of the brain and electroencephalogram were unremarkable. Subsequent investigations detected other micronutrient deficiencies (iron, copper, zinc) but negative coeliac and pernicious anaemia screens, bland cerebrospinal fluid, and negative serology for relevant infections and anti-neuronal antibodies. Magnetic resonance imaging (MRI) showed abnormal high T2 signal involving the dorsal column of the cervical cord (Fig. 1), consistent with subacute combined degeneration of the spinal cord.

On day 10 she was transferred to our rehabilitation unit. Dysarthria necessitating written communication aids persisted, despite intramuscular B12 replacement. Examination further identified profound tongue wasting with intermittent fasciculations and brisk jaw-jerk reflex. Limb reflexes were brisk and power was decreased in the left upper limb only. She had no sensory or gait abnormalities. Chronic paranoid delusions continued but were nondistressing. Her mini-mental state examination scored 27/ 30 points, and Frontal Assessment Battery 13/18 points, before discharge. The combination of bulbar weakness,

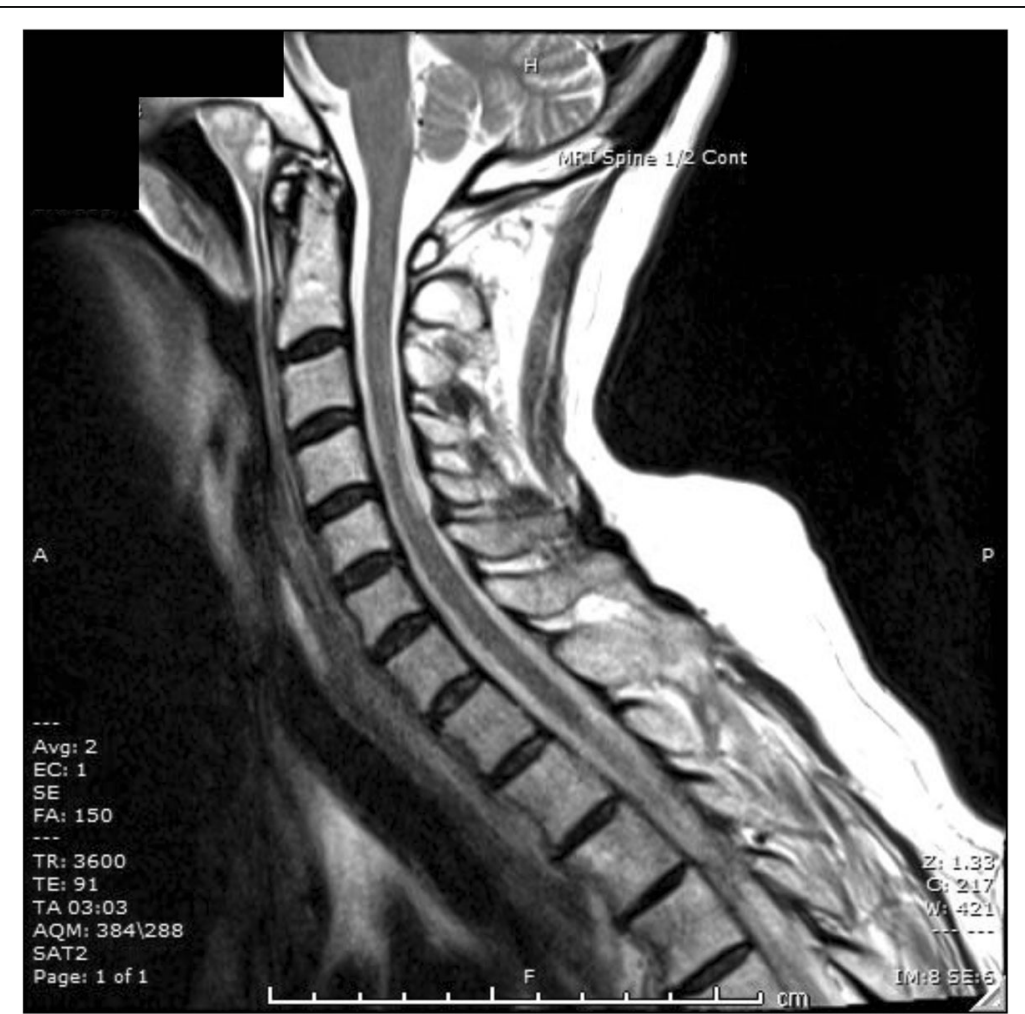

Fig. 1 T2-weighted magnetic resonance imaging (MRI) of the spine demonstrating abnormal high T2 signal involving the dorsal column of the cervical cord 
mixed upper and lower motor neuron signs and failure to improve despite B12 replacement prompted EMG. Widespread chronic denervation in upper and lower limbs and genioglossus alongside active denervation and fasciculation supported a diagnosis of MND. Interestingly, nerve conduction studies demonstrated no evidence of a sensorimotor neuropathy.

Our patient commenced treatment with riluzole and was referred to a multidisciplinary progressive neurological diseases service. Her disease progressed and she unfortunately died a number of months later.

\section{Discussion and conclusions}

Our case demonstrates several important clinical lessons. Firstly, the diagnostic difficulties posed by coexistence of vitamin B12 deficiency and another neurological pathology, particularly motor neuron disease (MND). Variability in neurocognitive manifestations of B12 deficiency can limit delineation of other pathologies clinically. Clinicians should also be aware of non-specific investigation findings including the limitations of imaging findings in the setting of B12 deficiency and motor neuron disease (MND). Secondly, we present a reminder of the importance of careful examination and re-assessment where co-morbid schizophrenia or other mental illness may cloud the initial diagnosis. To our knowledge, this is the first report highlighting this combination of diagnostic challenges.

Neurological and cognitive effects of B12 deficiency, including subacute combined degeneration of the spinal cord, are highly variable [6]. Dysarthria is atypical, however, and its prominence in our patient eventually triggered investigation for an alternate diagnosis. Prognosis is excellent if B12 deficiency is corrected, although neurological deficits may persist. In contrast, MND is a progressive disorder involving upper and lower motor neurons, often with prominence of bulbar weakness. It manifests as weakness in the limbs, bulbar and respiratory muscles, usually with asymmetrical onset. The diagnosis is clinical and may be supported by EMG findings. Sensory function, ocular muscles, continence and cognition are typically spared [2].

Although our patient was diagnosed with MND, initial proprioceptive disturbance which resolved following B12 replacement may reflect concurrent subacute combined degeneration. This may have been sequelae of her paranoid schizophrenia limiting her food repertoire, compounded by bulbar dysfunction affecting nutritional intake. We noted that previous vitamin B 12 levels had been normal.

Neuroimaging findings in both B12 deficiency and MND are non-specific and may be absent or very similar. Both conditions have been associated with MRI hyperintensity on T2 weighted images of the brain and cervical cord, with the anatomical distinction of the dorsal columns and the pyramidal tracts being typically abnormal in B12 deficiency and MND respectively $[1,6]$. However subacute combined degeneration of the spinal cord is documented to rarely result in demyelination of pyramidal and spinocerebellar tracts [7]. Another case report documented corticospinal tract hyperintensity on MRI scan of the brain of a B12-deficient patient whose neurocognitive symptoms improved with B12 replacement therapy, hence considered to be mimicking MND [4].

Clinically, vitamin B12 deficiency has been described to masquerade as antipsychotic-resistant psychosis [8]. Vitamin B12 supplementation with folate has been proposed as a method of improving negative symptoms in certain schizophrenic patient groups [9]. However a meta-analysis more recently reported that vitamin B12 levels in patients with schizophrenia were not lower, but rather higher, overall, than the control group [10]. The authors hypothesise that perhaps patients with chronic psychosis were receiving more frequent monitoring and correction of nutritional status.

A subset of MND occurs concurrently with frontotemporal dementia (FTD-MND) and may be initially misdiagnosed as a primary psychotic disorder. Its phenotype can include psychotic features, atypical MRI findings and, less commonly, primary progressive aphasia or parkinsonism [3]. The C9ORF72 mutation with hexanucleotide expansion is the commonest known associated gene in familial cases of FTD-MND and can occur sporadically. While her first-degree relative with schizophrenia is proposed to be an important predictive factor, our patient's history of over 30 years of antipsychoticresponsive symptoms preceding MND onset, however, is not in keeping with reported MND onset within 10 years of psychosis in FTD-MND [11]. Mutation testing for C9ORF72 was considered given her comorbid psychiatric diagnosis, however our patient deteriorated before testing could be discussed.

More recently, a genetic link between schizophrenia and ALS has been suggested by studies that have found the C9ORF72 expansion in first degree relatives with primary psychotic conditions without dementia and a significant genetic correlation between ALS and schizophrenia in a genome-wide study of over 100,000 people [12, 13]. This has increased interest in exploring overlapping management strategies for both conditions. The potential that neuroprotective effects of schizophrenia pharmacotherapy may protect against manifestations of ALS has also been hypothesised previously [14]. The relationship between neurodegenerative and neuropsychiatric conditions requires further delineation and characterisation.

Our case demonstrates the diagnostic challenges in the presence of concomitant and overlapping neurological conditions with co-existing psychiatric illness. The phenomenon of incorrectly attributing physical 
symptoms to a psychiatric condition is described in the literature as diagnostic overshadowing [5]. A number of case reports describe missed diagnoses due to comorbid psychosis $[15,16]$. To our knowledge, we report the first case of ALS/MND and vitamin B12 deficiency causing subacute combined degeneration in the context of schizophrenia. Other important factors delaying clinical diagnoses in patients with psychiatric conditions include communication difficulties, symptoms being attributed to medication side effects and delay in seeking medical attention [17, 18]. Clinical vigilance and re-evaluation are key aspects in facilitating accurate and timely diagnosis in this vulnerable cohort of patients.

\section{Abbreviations}

ALS: Amyotrophic lateral sclerosis; CT: Computed tomography; EMG: Electromyography; FTD: Frontotemporal dementia; MND: Motor neuron disease; MRI: Magnetic resonance imaging

\section{Acknowledgements}

Not applicable.

\section{Authors' contributions}

$\mathrm{KL}, \mathrm{AW}$ and $\mathrm{PL}$ were responsible for the clinical management of the patient. $\mathrm{PL}$ and AW were responsible for drafting and editing of the manuscript. KL and $\mathrm{W}$ participated in critical revision of the manuscript for intellectual content. All authors read and approved the final manuscript.

\section{Funding}

There are no sources of funding to declare.

\section{Availability of data and materials}

All the data supporting our findings is contained within manuscript.

\section{Ethics approval and consent to participate}

Ethics committee approval was not applicable as the information was analysed in a retrospective manner and had no effect on treatment.

\section{Consent for publication}

Written informed consent was obtained from the patient's next of kin for publication of this case report and any accompanying images. A copy of the written consent is available for review by the Editor-in-Chief of this journal.

\section{Competing interests}

The authors declare that they have no competing interests.

\section{Author details}

'Department of Medicine and Aged Care, The Royal Melbourne Hospital, Parkville, VIC, Australia. ${ }^{2}$ Department of Aged Care, Austin Hospital, Heidelberg, VIC, Australia. ${ }^{3}$ Department of Neurology, The Royal Melbourne Hospital, Parkville, VIC, Australia. ${ }^{4}$ University of Melbourne, Parkville, VIC, Australia.

Received: 21 July 2019 Accepted: 5 September 2019

Published online: 18 November 2019

\section{References}

1. Kalita J, Mista UK. Vitamin B12 deficiency neurological syndromes: correlation of clinical, MRI and cognitive evoked potential. J Neurol. 2008;255:353-9.

2. Brown Jnr RH. Chapter 452, Amyotrophic lateral sclerosis and other motor neuron diseases. In: Harrison's principles of internal medicine. 19th ed. New York: McGraw-Hill Education; 2015. p. 2631-6.

3. Devenney E, Hornberger M, Irish M, et al. Frontotemporal dementia associated with the C9ORF72 mutation, a unique clinical profile. JAMA Neurol. 2014;71(3):331-9.
4. Pinto W, de Souza P, Rogerio RM, et al. Vitamin B12 deficiency mimicking neuroimaging features of motor neuron disease [Translated from Spanish]. Arq Neuropsiquiatr. 2014;72(1):85.

5. Shefer G, Henderson C, Howard M, et al. Diagnostic Overshadowing and Other Challenges Involved in the Diagnostic Process of Patients with Mental IIIness Who Present in Emergency Departments with Physical Symptoms - A Qualitative Study. PLoS One. 2014;9(11):e111682.

6. Hemmer B, Glocker FX, Schumacher $M$, et al. Subacute combined degeneration: clinical, electrophysiological, and magnetic resonance imaging findings. J Neurol Neurosurg Psychiatry. 1998;65:822-7.

7. Katsaros VK, Glocker FX, Hemmer B, et al. MRI of spinal cord and brain lesions in subacute combined degeneration. Neuroradiology. 1998;40:716-9.

8. Raveendranathan D, Shiva A, Venkatasubramanian G, et al. Vitamin B12 Deficiency Masquerading as Clozapine Resistant Psychotic Symptoms in Schizophrenia. Lett J Neuropsychiatry Clin Neurosci. 2013;25:2.

9. Roffman JL, Lamberti JS, Achtyes E, et al. Randomized multicenter investigation of folate plus vitamin B12 supplementation in schizophrenia. JAMA Psychiatry. 2013;70(5):481-9.

10. Cao B, Wang DF, Liu YQ, et al. Vitamin B12 and the risk of schizophrenia: A meta-analysis. Schizophr Res. 2016;172:216-7.

11. Devenney EM, Landin-Romeroa $\mathrm{R}$, lrisha $\mathrm{M}$, et al. The neural correlates and clinical characteristics of psychosis in the frontotemporal dementia continuum and the C9orf72 expansion. Neurolmage-Clin. 2017;13:439-45.

12. Watson A, Pribadi M, Chowdari K, et al. C9orf72 repeat expansions that cause frontotemporal dementia are detectable among patients with psychosis. Psychiatry Res. 2016;235:200-2.

13. McLaughlin RL, Schijven D, van Rheenen W, et al. Genetic correlation between amyotrophic lateral sclerosis and schizophrenia. Nat Commun. 2017;8:e14774

14. Stommel EW, Graber D, Montanye J, et al. Does treating schizophrenia reduce the chances of developing amyotrophic lateral sclerosis? Med Hypotheses. 2007;69(5):1021-8.

15. Hu HC, Chiu NM. Delayed Diagnosis in an Elderly Schizophrenic Patient with Catatonic State and Pulmonary Embolism. Int J Gerontol. 2013;7:183e-185.

16. Retamero C, Paglia C. When patients do not hurt: silent acute abdomen in a patient with schizophrenia. Gen Hosp Psychiat. 2012;34(2):210.e9-11.

17. So R, Hirota T, Yamamoto $Y$, et al. Lack of cardinal symptoms of meningitis in a hospitalized patient with chronic schizophrenia: lessons to be learned. Gen Hosp Psychiatry. 2015;37(6):621.e3-4.

18. Farasatpour M, Janardhan R, Williams $C$, et al. Breast cancer in patients with schizophrenia. Am J Surg. 2013;206:798-804.

\section{Publisher's Note}

Springer Nature remains neutral with regard to jurisdictional claims in published maps and institutional affiliations.

\section{Ready to submit your research? Choose BMC and benefit from:}

- fast, convenient online submission

- thorough peer review by experienced researchers in your field

- rapid publication on acceptance

- support for research data, including large and complex data types

- gold Open Access which fosters wider collaboration and increased citations

- maximum visibility for your research: over $100 \mathrm{M}$ website views per year

At BMC, research is always in progress.

Learn more biomedcentral.com/submission 